\title{
Direct toroidal torque driven by ICRF heating and its dependence on the plasma rotation
}

\author{
R. Bilato, M. Brambilla, E. Fable, E. Poli \\ Max Planck Institute for Plasma Physics, EURATOM Association, Boltzmannstr. 2, \\ 85748 Garching, Germany \\ E-mail: roberto(dot)bilato (at) ipp (dot)mpg (dot)de
}

\begin{abstract}
The contribution of ICRF waves as source of direct external toroidal torque is investigated and shown to be too small to be the main mechanism behind the common experimental observations of plasma rotation in the presence of ICRFheating. In particular, the impact of plasma rotation on the direct ICRF torque is addressed with the outcome that plasma rotation mainly influences the profiles of the ICRF torque and not its total value.
\end{abstract}

\section{Introduction}

On many fusion devices an appreciable variation of the profiles of the plasma toroidal velocity appears in response to the heating of a minority species with radio-frequency $(\mathrm{RF})$ waves in the ion cyclotron (IC) range of frequencies (ICRF). Due to its peculiar feature of increasing mainly the perpendicular (with respect to direction of the confining magnetic field, B) kinetic energy of the resonating ions, ICRF augments the flow of passing particles towards the trapped region in velocity space. In becoming trapped, particles experience radial drifts of their guiding centers that establish a radial current, which, in turn, causes an opposite balancing radial component of the plasma current $\mathbf{j}$. The latter is responsible for a $\mathbf{j} \times \mathbf{B}$ toroidal torque on the plasma (when not clearly stated, hereafter we refer to the toroidal component of the torque). In the limit of zero-banana width approximation, on each magnetic surface this torque is compensated by the collisional friction of fast particles slowing-down on the bulk species [1], and the total toroidal torque is equal to the external one due to ICRF waves absorbed on that magnetic surface. However, still in compliance with the conservation of total angular momentum, finite-banana width effects can be responsible for sheared torques which can be strong enough to affect the plasma confinement. Therefore, by influencing the plasma confinement as heating source and by inducing sheared flows, ICRF can have an intrinsic/indirect impact on the toroidal momentum balance $[2,3,4,5]$. In addition, ICRF waves can exert an external/direct torque mainly in two ways. The first is associated with the toroidal momentum carried by the waves, which is given up to the plasma when they are absorbed. The toroidal momentum carried by waves is 
small, as explained in due course, and it is further reduced when the spectrum of the antenna currents is symmetric in the toroidal wavenumber, $n_{\varphi}$ (hereafter this setup is called ICRF-heating mode). The second mechanism of external torque is the increase of the rate of prompt and ripple losses caused by ICRF acceleration. This torque is in counter-current direction, since the particles that exit the plasma are moving along the outer leg of their banana orbits with co-current toroidal velocity [6]. The impact of ICRF heating on plasma toroidal momentum is an ample and complex topic. For an exhaustive overview of the present understanding we refer to $[7,8]$ and the references therein.

Here, our interest is focused mainly on the recent work by $\mathrm{Xu}$ and co-workers where they pointed out that in rotating plasmas there is a net torque exerted by RF waves, even when operating in ICRF-heating mode [9]. The mechanism is based on the Doppler shift associated with the plasma rotation, which breaks the $n_{\varphi^{-}}$-symmetry in the wave absorption and, as a consequence, in the transfer of momentum from waves to the plasma. In their clear and motivating article [9], Xu and co-workers draw conclusions implicitly subject to the assumption that only a part of the launched ICRF spectrum is absorbed by the plasma. However, one expects that also the other part of the ICRF spectrum is eventually absorbed by the plasma, otherwise the mechanism proposed in [9] would occur at the price of a drastic reduction of the heating efficiency. Therefore, under the reasonable assumption that almost all the coupled RF power is eventually absorbed by the plasma, the Doppler shift can have an impact on the profile of the torque density, but momentum conservation implies that the total applied torque can be only slightly influenced by the plasma rotation. As quantitative examples, in section 2 we estimate with the TORIC full-wave code [10] the maximum toroidal torque per absorbed power that ICRF-heating can directly exert on the plasma. Moreover, it is shown that the torque exerted by ICRF is very small, even under the assumption that the ICRF power is carried by a single toroidal wave mode. In section 3 , by accounting for ICRF-heating as the only external source in the angular momentum balance equation, we quantify the order-of-magnitude of the impact of the ICRF toroidal torque on the plasma rotation. For typical plasmas of medium-size experiments like ASDEX-Upgrade (AUG), we show that this is a small effect, since the toroidal momentum carried by waves is much smaller that that carried by neutrals of the neutral beam injection (NBI) system $[11,12]$. This is not in contradiction with the common experimental finding that ICRF heating can substantially influence plasma rotation $[8,13]$. In fact, ICRF heating modifies the plasma pressure profiles, influences turbulence and MHD activity. This indirect impact of ICRF heating on plasma rotation, however, is a much more complex process, and not considered in the present work. Finally, to address a possible increase of the external torque exerted by ICRF heating when the plasma is already rotating, in section 4 we approximate the distribution function of the resonating species by a shifted-Maxwellian [14,9]. Contrary to what predicted in [9], we find that the impact of the plasma rotation on the enhancement of the ICRF torque is negligible for a wide range of plasma velocities. 


\section{RF direct torque}

A precise calculation of the toroidal momentum transfer from waves to plasma in the kinetic equation requires to complement the gyro-averaged quasilinear operator with the terms that depend on the gyro-phase [15]. In the following we discuss a simplified derivation of equation (18) in [15] based on the assumption that waves transfer their toroidal momentum to the plasma at the location where they are absorbed.

In a toroidal system it is natural to expand the wave fields in toroidal eigenmodes, $\exp \left\{\operatorname{in} n_{\varphi}\right\}$, with $\varphi$ the toroidal angle and $n_{\varphi}$ the corresponding eigenvalue, known as toroidal wave (or mode) number. In other words, $n_{\varphi}$ is the toroidal covariant component of the wavevector, $\mathbf{k}$, whose toroidal physical component is $k_{\varphi}=n_{\varphi} / R$, with $R$ the distance from the torus axis. The toroidal momentum carried by a photon associated with the toroidal component $n_{\varphi}$ of the wave field is $p_{\varphi}=\hbar k_{\varphi}$, with $\hbar$ the reduced Planck constant. Therefore, if $\dot{n}_{\omega}$ is the injection rate of photons, the torque exerted, $T_{\varphi}=R \hbar k_{\varphi} \dot{n}_{\omega}=\hbar n_{\varphi} \dot{n}_{\omega}$, depends only on $n_{\varphi}$. By observing that $\dot{n}_{\omega}=P_{\mathrm{ICRF}} / \hbar \omega$ with $\hbar \omega$ the photon energy, $\omega$ the angular frequency of the waves, and $P_{\text {ICRF }}$ the coupled ICRF power, the total ICRF toroidal torque is $T_{\mathrm{ICRF}}=\left(n_{\varphi} / \omega\right) P_{\mathrm{ICRF}}$, with the ratio $n_{\varphi} / \omega$ playing the role of torque efficiency. In an axisymmetric plasmas, the power carried by each toroidal mode $n_{\varphi}$ can be absorbed by the plasma but not transferred to other toroidal modes. As a consequence, in axisymmetric plasmas the ICRF toroidal angular momentum is conserved and eventually transferred to the plasma by absorption.

For the sake of comparison with NBI, the toroidal torque density due to NBI heating can be written as $\mathcal{T}_{\mathrm{NBI}}(r)=m_{i}\left\langle v_{\varphi, \mathrm{b}}(r, \vartheta) R_{\mathrm{b}}(r, \vartheta) \dot{n}_{\mathrm{b}}(r, \vartheta)\right\rangle$, with $R_{\mathrm{b}}$ the distance from the torus axis of the birth location, $v_{\varphi, \mathrm{b}}$ the toroidal component of the velocity at the birth position, $\dot{n}_{\mathrm{b}}(r)$ the local birth rate per unit volume [1], and $\langle\cdots\rangle$ the surface average. In the case of NBI, it is the linear momentum of the injected neutrals that is conserved and eventually transferred to the the plasma when they are ionized. Therefore, the total exerted torque depends on the ionization profiles of the fast injected neutrals. With the aim of deriving a rough order-of-magnitude comparison between ICRF and NBI, we circumvent the surface-average operation by approximating the total NBI torque with $T_{\mathrm{NBI}} \approx R_{a}\left(\cos \left(\varphi_{\mathrm{inj}}\right) / v_{\mathrm{inj}}\right) P_{\mathrm{NBI}}$, with $\varphi_{\text {inj }}$ the toroidal injection angle, $v_{\text {inj }}$ the injection speed and $R_{a}$ the magnetic-axis radius. To derive $T_{\mathrm{NBI}}$ we used the fact that the total injection rate, $\dot{n}_{\text {inj }}$, which determines $\dot{n}_{b}$, can be written in terms of the total injected power, $P_{\mathrm{NBI}}=m_{i} v_{\mathrm{inj}}^{2} \dot{n}_{\mathrm{inj}} / 2$. It follows that

$$
\frac{T_{\mathrm{ICRF}}}{T_{\mathrm{NBI}}} \approx\left(\frac{1}{k_{0} R_{a}} \frac{n_{\varphi}}{\cos \left(\varphi_{\mathrm{inj}}\right)}\right) \frac{v_{\mathrm{inj}}}{c} \frac{P_{\mathrm{ICRF}}}{P_{\mathrm{NBI}}},
$$

with $k_{0}=\omega / c$. The ratio $n_{\varphi} /\left(k_{0} R_{a}\right)$ is typically about $0.1 \div 0.2$ for the ICRF heating configuration in present devices (in $\mathrm{AUG}, n_{\varphi} \approx 12$, the ICRF frequency is around $30 \mathrm{MHz}$, and $R_{a}=1.6 \mathrm{~m}$ ). Thus, the factor in parenthesis is of the order of the unity for typical NBI launch geometries. On the contrary, the injection velocity normalized to the light speed is very small, e.g. about 0.007 for a $90 \mathrm{keV}$ deuterium beam. Since 
it is technically challenging to generate an ICRF toroidal spectrum with the main peak at very high $n_{\varphi}$ values and with high directionality (e.g. current-drive configuration) to avoid compensations between modes of different $n_{\varphi}$ sign, in realistic conditions and for the same absorbed power the ICRF torque is easily orders of magnitude smaller than the NBI one. Nevertheless, for completeness in the following we quantify it for AUG-like discharges, and estimate its impact on the plasma rotation.

The expression of $T_{\mathrm{ICRF}}$ in terms of $P_{\mathrm{ICRF}}$ can be extended to the ICRF toroidal torque density, $\mathcal{T}_{\varphi}$

$$
\mathcal{T}_{\varphi}(r)=\sum_{n_{\varphi}} \mathcal{T}_{n_{\varphi}}(r)=\sum_{n_{\varphi}} \frac{n_{\varphi}}{\omega} \mathcal{P}_{n_{\varphi}}(r),
$$

where $\mathcal{P}_{n_{\varphi}}$ is radial profile of $\mathrm{RF}$ absorbed power density corresponding to the toroidal wave number $n_{\varphi}$. The sum is over the toroidal modes excited by the antenna. By means of (2) the net wave torque can be calculated directly from the deposition profiles, $\mathcal{P}_{n_{\varphi}}$, estimated with ICRF codes $[16,17,18,19,20,21]$. In the TORIC code, the evaluation is done in the same way as done for the evaluation of the driven current with the EhstKarney parametrization [22] for the current-drive efficiency [23, 24].

In the case of ICRF-heating, the RF power eventually absorbed by the plasma does not necessarily inherit the $n_{\varphi}$-symmetry of the antenna currents, $P_{-n_{\varphi}} \neq P_{n_{\varphi}}$. The break of symmetry is due to the poloidal component of the confining magnetic field. A net toroidal momentum transfer from waves to the plasma can be expected also in the case of spectra which are perfectly symmetric in $n_{\varphi}$, as it is in the ICRF-heating mode. The aim is, therefore, to quantify this direct torque for realistic plasma and antenna parameters to infer its importance in influencing plasma rotation.

For the sake of realism in calculating the ICRF torque, as reference profiles we consider the equilibria and plasma profiles of three AUG discharges, designed to investigate plasma rotation in the presence of ICRF heating only [6], without, however, the aim here to analyze them. In these discharges the RF frequency is $36.5 \mathrm{MHz}$ and the magnetic field on axis is $B_{0}=2.48,2.74,2.29 \mathrm{~T}$, respectively for AUG26620, AUG26621, and AUG26622. The ICRF scheme is hydrogen minority (about 5\%) ICfundamental heating in deuterium plasmas in the absence of NBI and with about $5 \mathrm{MW}$ of RF coupled power. The corresponding position of the fundamental IC resonance of hydrogen is shown in figure 1.left. To reduce the differences between these discharges, in the simulations the density and temperature profiles are assumed the same and equal to those of AUG26621 at $4.8 \mathrm{sec}$ (figure 1.right). Where not specified, in the following it is assumed $5 \%$ of $\mathrm{H}$ in deuterium plasmas. Simulations of these discharges with TORIC-SSFPQL [26, 32], done under the assumption that the coupled RF power is completely absorbed by the plasma, show that close to the peak of ICRF absorption ( $\rho \approx 0.4$ on the LFS for AUG26621, and $\rho \approx 0.1,0.3$ on the HFS for AUG26620 and AUG26622, respectively) the average energy of ICRF-heated protons is about 50, 96 and $76 \mathrm{keV}$ for AUG26621, AUG26620, and AUG26622, respectively. Because of the zero-banana-width assumption in SSFPQL equations, these values are 

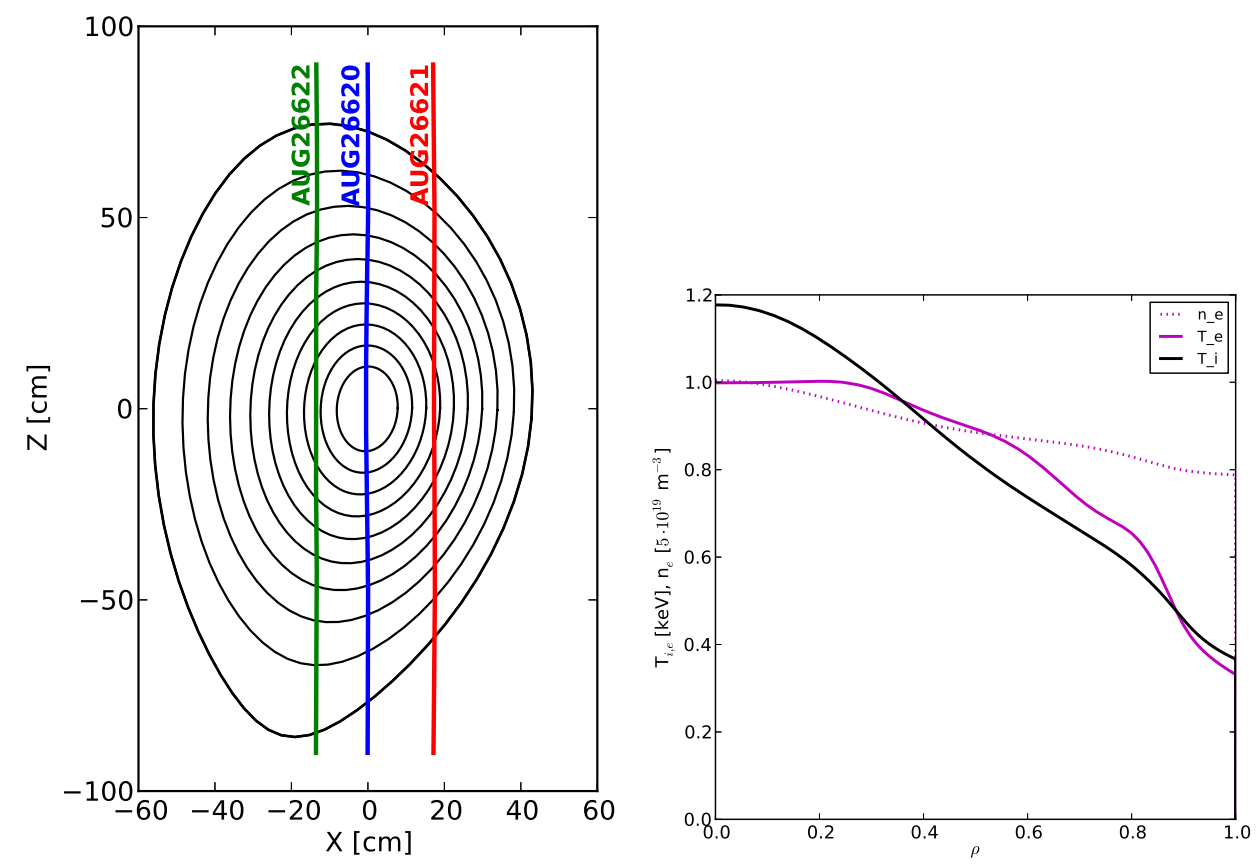

Figure 1. Left: Position of the ion-cyclotron resonance of hydrogen minority for the considered discharges; right: Plasma profiles used in the simulations.

somewhat overestimated. At these average energies the radial widths of the banana orbits with their tips roughly on the IC resonance are approximately 10, 15 and $13 \mathrm{~cm}$ for AUG26621, AUG26620, and AUG26622, respectively, and they are much smaller than the distance of the banana centers from the plasma edge. As an example of the the radial profiles of the torque density, figure 2.left shows these profiles for $n_{\varphi}= \pm 12$, which are the representative $n_{\varphi}$ values of the main peaks of the ICRF launched spectrum in AUG. Figure 2.right shows the integrated torque for the same profiles of figure 2.left Since they are not symmetric, their superimposition does not give a zero local torque density, but the amplitude of the total torque is in absolute value the same in all these cases since $\left|n_{\varphi}\right|$ is the same.

To evaluate the total torque, it is necessary to consider the whole launched spectrum, which is shown in figure 3 for the three cases under consideration. The difference in the magnetic field, and thus in the IC resonance position between the three cases, produces slight differences in the power spectra. The local peaks in the spectra are due to the so-called "cavity modes", e.g. weakly damped eigenmodes between the R-cutoff at the plasma edge and ion-ion cutoff in the plasma. Therefore, these peaks are sensitive to the position of the cutoffs, which in turn depends on the plasma profiles, $\omega$ and $n_{\varphi}$. The profiles of the total torque density, shown in figure 4 , are obtained by summing over $n_{\varphi}$ with the weights given by the the launched power spectra of figure 3. The comparison between figures 2 and 4 points to the importance of accounting for the whole antenna spectrum in evaluating the profile of the total torque density. The profiles in figure 4 are different from the profiles in figure 2 both in shape and, especially, in the amplitude, 

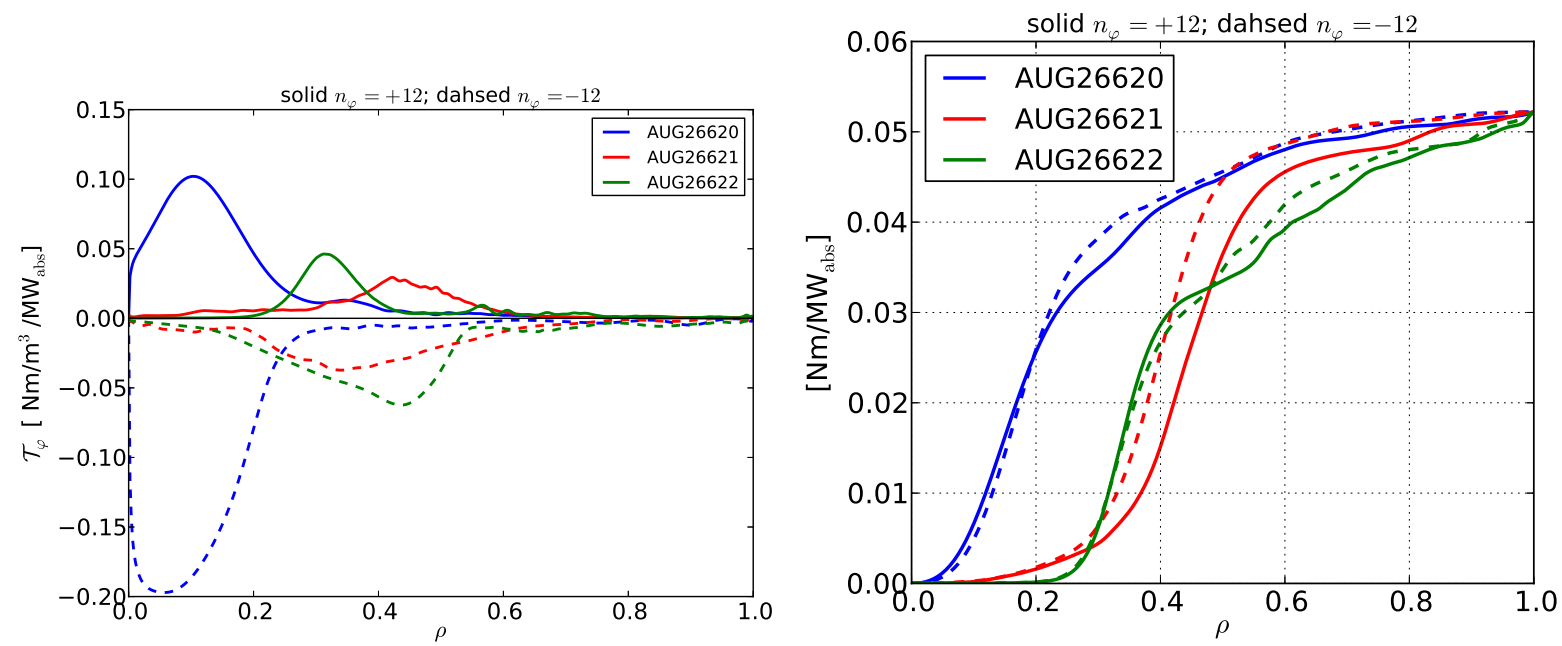

Figure 2. Left: Profiles of the torque density when the power is assumed to be carried by a single mode, which is 12 and -12 for solid and dashed lines, respectively. Because of its definition (2), the profile of $\mathcal{T}_{\varphi}$ corresponding to each $n_{\varphi}$ is proportional to the profiles of the absorbed power density, $\mathcal{P}_{\varphi}$. Right: The integrated torque for the same cases (the dashed lines are shown with the opposite sign to ease the comparison with the solid ones).
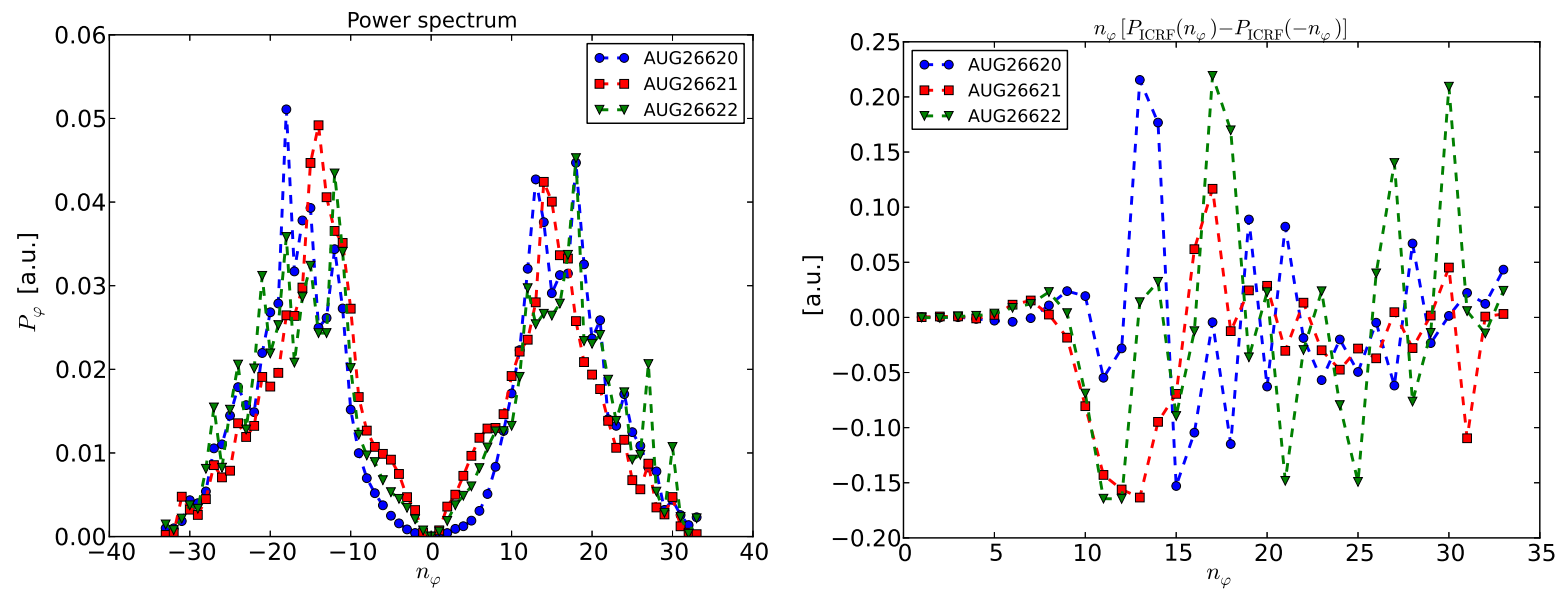

Figure 3. Left: Antenna spectra calculated by TORIC. The peaks are the footprints of "cavity modes". Right: Difference $n_{\varphi}\left[P_{\varphi}\left(n_{\varphi}\right)-P_{\varphi}\left(-n_{\varphi}\right)\right]$.

at least one order-of-magnitude smaller as a consequence of a compensation between $n_{\varphi}$ of opposite sign.

\section{ICRF direct contribution to the plasma toroidal velocity}

Especially in the minority heating scheme, the ICRF-accelerated ions can be treated as small population, and the plasma can be described as two interacting parts, namely the bulk plasma species and the ICRF-accelerated fast ions. Then the toroidal momentum equation for the toroidal plasma velocity $V_{\varphi}$, averaged over a magnetic surface, can be 


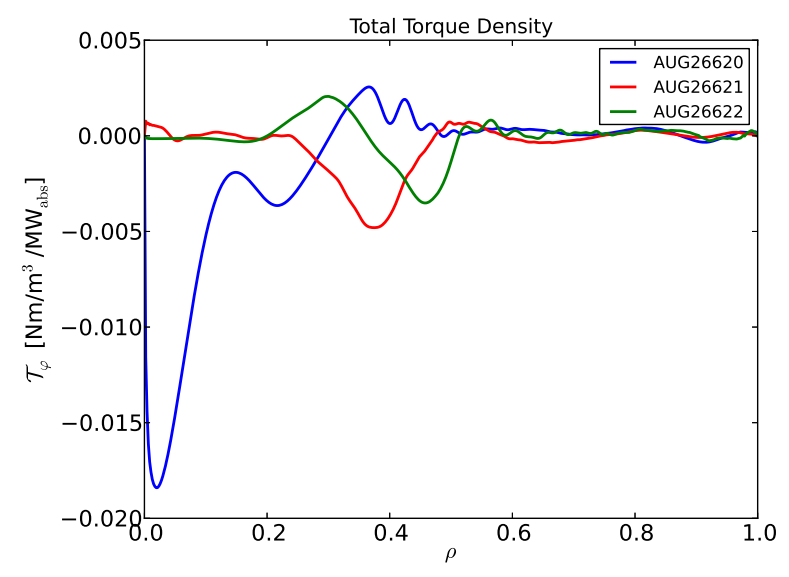

Figure 4. Profile of the torque density when the whole wave spectrum (see figure 3) is taken into account.

written as $[1,12,25]$,

$$
m_{i} n_{i} \frac{\partial}{\partial t}\left\langle R V_{\varphi}\right\rangle+\left\langle R \hat{e}_{\varphi} \cdot\left(\nabla \cdot \Pi_{i}\right)\right\rangle=\mathcal{T}_{\varphi}
$$

The ICRF direct contribution is $\mathcal{T}_{\varphi}$ of $(2)$. In steady state, the toroidal torque applied by ICRF heating is re-distributed by the ion-viscous stress tensor $\boldsymbol{\Pi}_{i}$, which is here approximated with a diffusive model, characterized by the diffusion coefficient $D$,

$$
\frac{\mathcal{T}_{\varphi}}{n_{i} m_{i}}+\frac{1}{\rho} \frac{\partial}{\partial \rho}\left\{\rho D(\rho) \frac{\partial\left\langle R V_{\varphi}\right\rangle}{\partial \rho}\right\}=0
$$

For a rough estimate of the impact of $\mathcal{T}_{\varphi}$ on $V_{\varphi}$ we assume $D(\rho)$ linear in $\rho$ with typical values for AUG, namely $D(0)=0.5 \mathrm{~m}^{2} / \mathrm{s}$ and $D(0.8)=2 \mathrm{~m}^{2} / \mathrm{s}$. By integrating twice equation (4), one can calculate the profiles of $\Delta U_{\varphi}(\rho):=\left(\left\langle R V_{\varphi}\right\rangle_{\rho}-\left\langle R V_{\varphi}\right\rangle_{\rho=1}\right) /\langle R\rangle$,

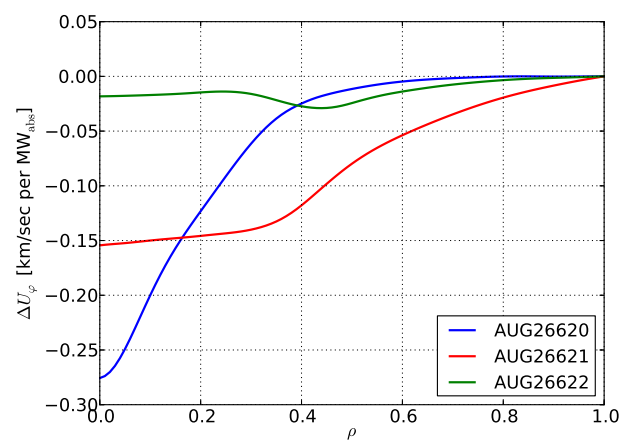

Figure 5. Profiles of $\Delta U_{\varphi}$ due to the toroidal RF-driven torque $\mathcal{T}_{\varphi}$ of figure 4.

which is a proxy of the $V_{\varphi}$ change, $\Delta V_{\varphi}$. Figure 5 shows $\Delta U_{\varphi}$ for $\mathcal{T}_{\varphi}$ of figure 4 for $1 \mathrm{MW}$ of ICRF absorbed power. Even for $5 \mathrm{MW}$ of RF power in these discharges, the values of $\Delta U_{\varphi}$ are much smaller than the experimental values of $V_{\varphi}$, which in these discharges are about a few $\mathrm{km} / \mathrm{s}[6]$. We conclude that the impact of the direct ICRF torque, $\mathcal{T}_{\varphi}$, on $V_{\varphi}$ is negligible on the background plasma velocity. 


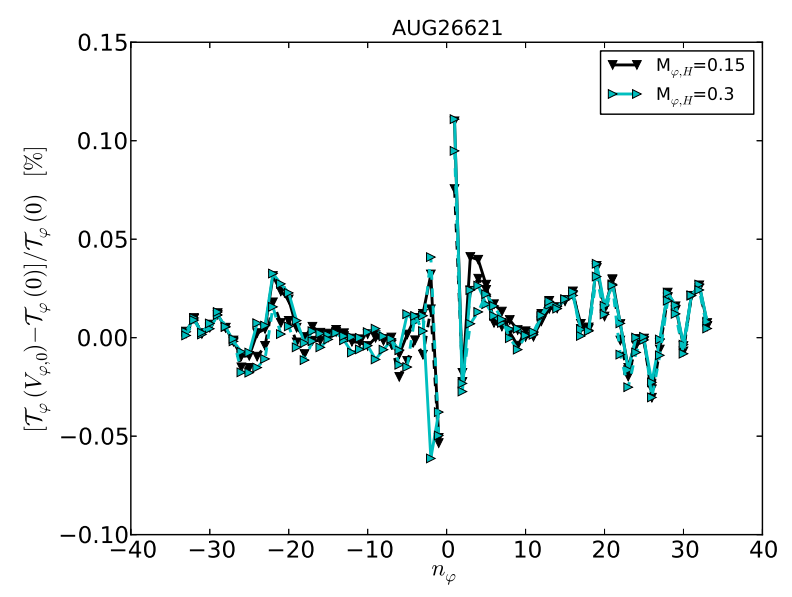

Figure 6. Relative change of the total toroidal torque as function of the toroidal wavenumber when the plasma toroidal velocity is varied. Solid and dashed lines join the points of positive and negative $V_{\varphi}$, respectively.

\section{Impact of plasma rotation on wave absorption and toroidal torque}

When a plasma rotates with a toroidal velocity $V_{\varphi, 0}$ with respect to the laboratory frame of reference, the absorption of RF waves is modified by the associated Doppler shift, which is proportional to $n_{\varphi} V_{\varphi, 0} / R \omega$. In particular, since the Doppler shift depends on the sign of $n_{\varphi}$, plasma rotation can be a source of asymmetry in the exerted torque [9]. The TORIC code accepts generic distribution function, as explained in [26, 27, 28], and we have considered a shifted-Maxwellian in order to test the mechanism proposed by $\mathrm{Xu}$ and co-workers [9], based on the Doppler shift associated with the plasma rotation,

$$
f\left(v_{\perp}, v_{\|}\right)=n(\rho)\left(\frac{m}{2 \pi T}\right)^{3 / 2} \exp \left\{\frac{m}{2 T}\left(\mathbf{v}-\mathbf{V}_{\varphi, 0}\right)^{2}\right\} .
$$

Strictly speaking, in rotating plasmas, the density is poloidally inhomogeneous as reaction to the centrifugal force [14]. To the poloidal inhomogeneity of the density can contribute also fast ions generated by NBI and/or ICRF heating [29]. For simplicity, here we do not account for the poloidal dependence of the density in TORIC, since, as it will be clear in the following, the effects of the plasma rotation on the ICRF torque are anyway negligible.

To quantify the impact of the plasma rotation on the capability of ICRF-heating to exert a torque, we have considered the off-axis heating case AUG26621 and assumed the hydrogen Mach number $M_{\varphi, H}=V_{\varphi, 0} / v_{\text {thH }}(\rho)$ radially constant, with $v_{\text {th }}=\sqrt{2 T_{H}(\rho) / m_{H}}$ the hydrogen thermal speed. We explore $M_{\varphi, H}$ equal to \pm 0.15 and \pm 0.3 , largely covering the maximum range of plasma rotation in the AUG plasma core, even in NBI plasmas [11]. Indeed, $M_{\varphi, H}$ corresponding to intrinsic rotation without NBI heating is definitively lower than 0.1 in AUG [30]. The profile of the corresponding Mach number of an ion species $i$ is $M_{\varphi, i}=M_{\varphi, H} v_{\text {thH }}(\rho) / v_{\text {thi }}(\rho)=M_{\varphi, H} \sqrt{m_{i} / m_{H}}$, where the latter equality holds for equal ion temperatures, as in our cases. The impact of the 

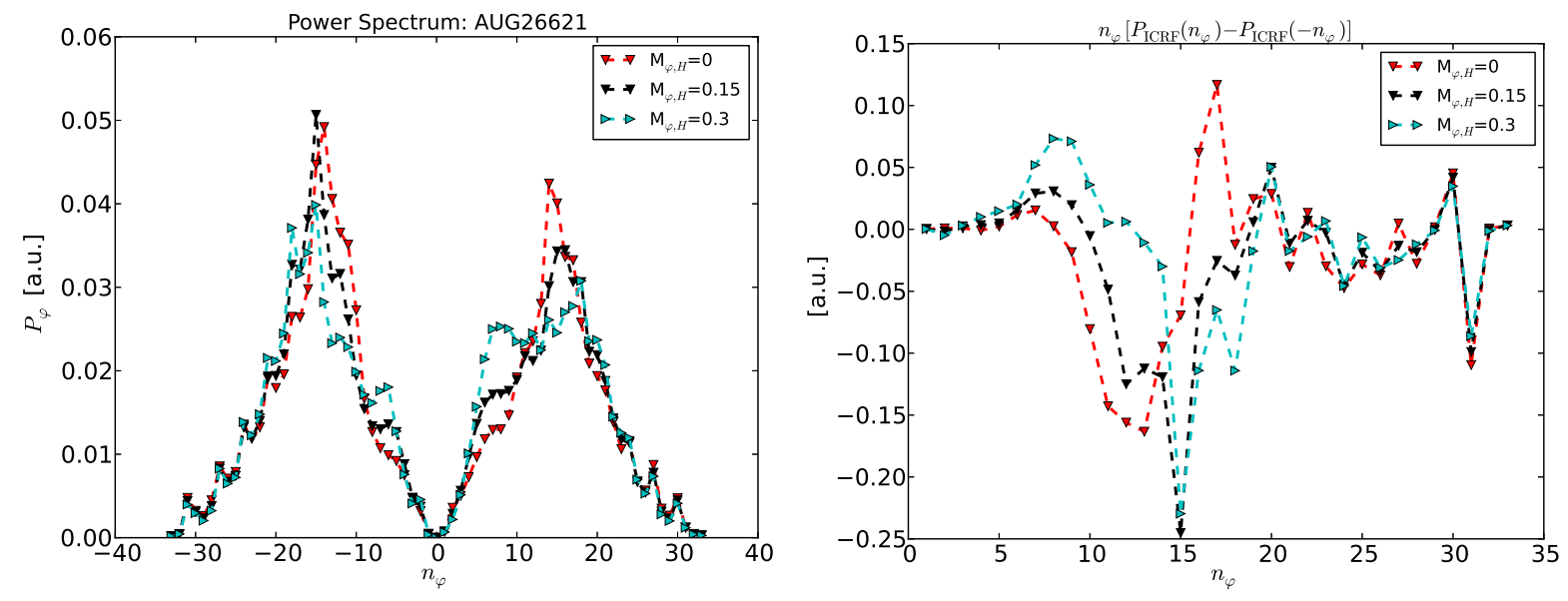

Figure 7. Left: Antenna spectra calculated by TORIC for different values of the plasma toroidal velocity. Right: Difference $n_{\varphi}\left[P_{\varphi}\left(n_{\varphi}\right)-P_{\varphi}\left(-n_{\varphi}\right)\right]$.

plasma rotation on the $\mathrm{RF}$ torque is negligible, as it can be seen in figure 6. By contrast, the plasma rotation has an appreciable impact on the power spectrum, figure 7 , and consequently on the total torque density, figure 8. The corresponding $\Delta U_{\varphi}$ profiles are

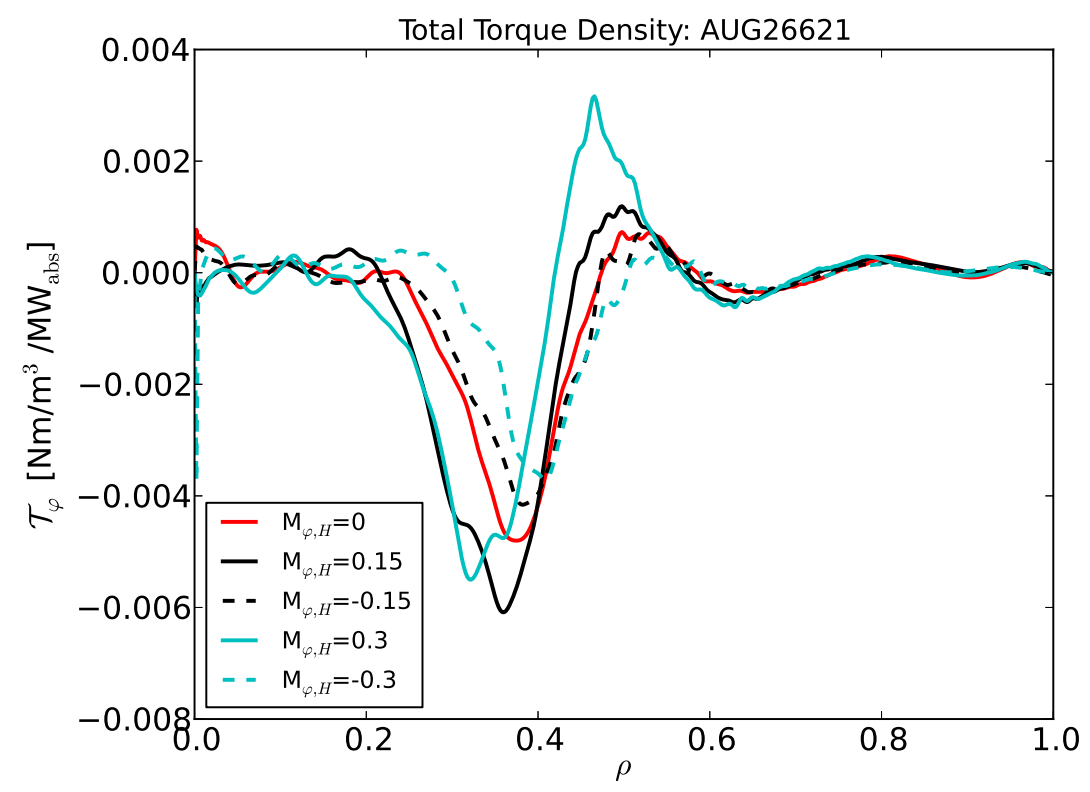

Figure 8. Profile of the torque density for different plasma velocities. The reference case in the absence of plasma rotation of figure 4 is the solid red line.

shown in figure 9. Plasma rotation shifts and deforms the ICRF absorption profiles, but obviously does not prevent the ICRF power to be absorbed completely, contrary to what implicitly assumed in [9]. Although plasma rotation influences the torque profiles by deforming the absorbed power profiles, the total torque is always negligible, since the toroidal momentum carried by RF waves is very small, especially in comparison with the one induced by NBI beams. 


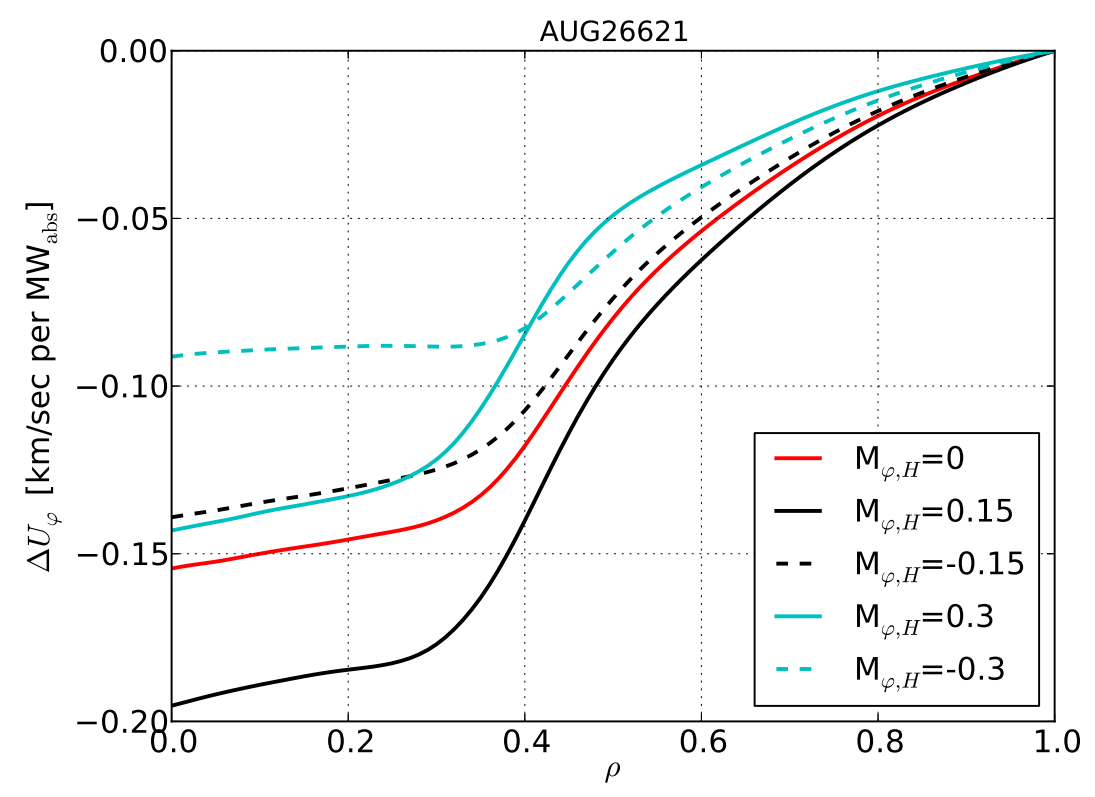

Figure 9. Profiles of $\Delta U_{\varphi}$ due to the toroidal RF-driven torque $\mathcal{T}_{\varphi}$ shown in figure 8.

So far, TORIC and the equation (4) are linear in the ICRF absorbed power. Strictly

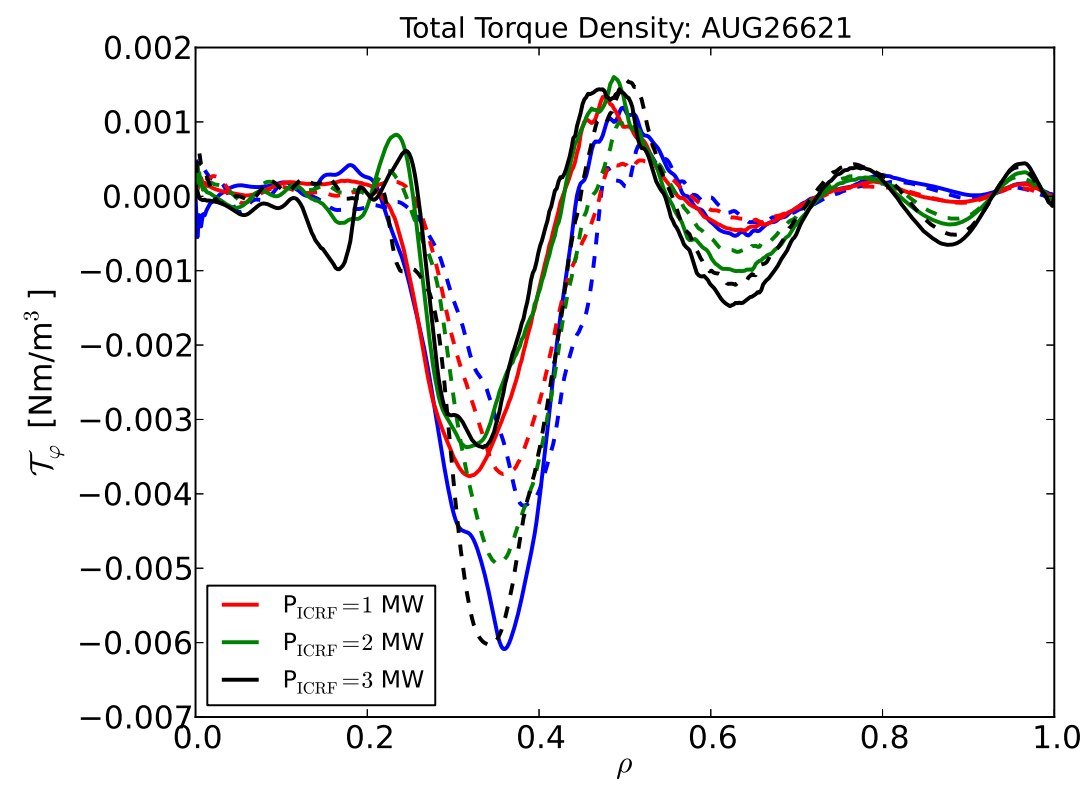

Figure 10. Profiles the toroidal torque density for $M_{\varphi, H}=0.15$ (solid) and $M_{\varphi, H}=-0.15$ (dashed). The blue lines show the torque density calculated by TORIC with the bi-Maxwellian (5) as distribution functions, whereas the red (1 MW), the green $(2 \mathrm{MW})$, and the magenta (3 MW) with the numeric distribution function of SSFPQL at the convergence of the TORIC-SSFPQL loop.

speaking, the back-reaction of ICRF heating on the wave propagation and absorption makes the set of wave and kinetic equations nonlinear in the ICRF power. The package TORIC-SSFPQL can take this into account, since it solves this set of equations 


\begin{tabular}{|c|c|c|c|}
$\begin{array}{c}P_{\text {ICRF }} \\
{[\mathrm{MW}]}\end{array}$ & $M_{\varphi, H}$ & Dist. Func. & $\begin{array}{c}T_{\varphi} \\
{[\mathrm{Nm}]}\end{array}$ \\
\hline 1 & 0.15 & bi-Maxw & -0.0035 \\
1 & 0.15 & SSFPQL & -0.0018 \\
2 & 0.15 & SSFPQL & -0.0026 \\
3 & 0.15 & SSFPQL & -0.0032 \\
\hline 1 & -0.15 & bi-Maxw & -0.0029 \\
1 & -0.15 & SSFPQL & -0.0026 \\
2 & -0.15 & SSFPQL & -0.0039 \\
3 & -0.15 & SSFPQL & -0.0048
\end{tabular}

Table 1. Values of the total toroidal torque for the profiles of figure (10).

selfconsistently [26, 31]. As an example, figure 10 shows the torque density profiles for $M_{\varphi, H}=0.15$ (solid and dashed lines for positive and negative $M_{\varphi, H}$, respectively) for $1 \mathrm{MW}$ (red), $2 \mathrm{MW}$ (green), and $3 \mathrm{MW}$ (black) of ICRF absorbed power. As reference, the blue lines are the torque density profiles with the initial bi-Maxwellian (5), which is the initial step of the TORIC-SSFPQL iteration loop [26]. The nonlinearities of the set of wave and kinetic equations have visible effects on the shape of $\mathcal{T}_{\varphi}$, but they do not boost the contribution of the total torque $T_{\varphi}$, as reported in the table 1. Since SSFPQL assumes zero-width orbits [32], the additional contributions to the torque analyzed in $[2,3]$ cannot unfortunately be addressed here.

\section{Conclusions}

The radial profiles of the ICRF toroidal torque density have been calculated by using (2) for the torque density and the ICRF power density profiles calculated by the full-wave TORIC code, in the same way as done for the driven current [23]. The total torque per MW of absorbed power is very small when compared with the typical values of the torque associated with the NBI heating. The estimated variations of the plasma toroidal velocity directly induced by the ICRF external torque are at least an order of magnitude smaller than the typically measured plasma velocities, even when one assumes that the RF power is carried by a single and optimal toroidal wavenumber. In addition, the Doppler shift associated with the plasma rotation influences the radial profiles of the toroidal torque and only slightly the values of the total toroidal torque. Indeed, the applications to AUG-like plasmas considered here point out that ICRFheating plays a minor role as external source of total toroidal torque even in rotating plasma. Contrary to what claimed in [9], this holds also for ITER where the more ICRF available power is more than compensated by the larger plasma inertia due to its larger volume. Nevertheless, although the torque due to wave momentum transfer to the plasma is negligible, ICRF heating can have a relevant impact on the plasma rotation by modifying the plasma pressure, by influencing turbulence and MHD activity, as observed 
experimentally.

\section{Acknowledgment}

We would like to acknowledge the interesting discussions on this topic had with C. Angioni, K. Lackner, O. Maj, and Y. Podoba. We are indebted to anonymous referees for providing insightful and useful comments.

[1] Hinton, F. and ROSEnbluth, M., Physics Letters A 259 (1999) 267.

[2] PERkins, F. W., WHIte, R. B., BONOLI, P. T., and CHAN, V. S., Physics of Plasmas 8 (2001) 2181.

[3] ERIKSSON, L.-G. and PORCELLI, F., Nuclear Fusion 42 (2002) 959.

[4] PARRA, F. I. and BARNES, M., Plasma Physics and Controlled Fusion 57 (2015) 045002.

[5] LAZZARO, E., NOWAK, S., and SAUTER, O., Plasma Physics and Controlled Fusion 58 (2016) 124003.

[6] POdobA, Y., ANGIOni, C., BILATO, R., et al., in 39th EPS Conference on Plasma Physics, p. P4.090, EPS, 2012.

[7] ERIKSSON, L.-G., Hellsten, T., NAVE, M. F. F., et al., Plasma Physics and Controlled Fusion 51 (2009) 044008.

[8] RICE, J. E., Plasma Physics and Controlled Fusion 58 (2016) 083001.

[9] XU, Y., WANG, S., and ZHANG, D., Nuclear Fusion 56 (2016) 046013.

[10] BRAmBiLla, M., Plasma Physics and Controlled Fusion 41 (1999) 1.

[11] MCDERMotT, R. M., ANGiOni, C., DUX, R., et al., Plasma Physics and Controlled Fusion 53 (2011) 124013.

[12] Solomon, W. M., BURrell, K. H., GAROFAlO, A. M., et al., Physics of Plasmas 17 (2010).

[13] MANTSinen, M. J., BILATO, R., BOBKOV, V. V., et al., AIP Conference Proceedings 1689 (2015).

[14] HINTON, F. L. and WONG, S. K., Physics of Fluids 28 (1985) 3082.

[15] LEE, J., PARRA, F. I., PARKER, R. R., and BONOLI, P. T., Plasma Physics and Controlled Fusion 54 (2012) 125005.

[16] BILATO, R., BERTELli, N., BRAMBILlA, M., et al., AIP Conference Proceedings 1689 (2015) 060001.

[17] LAMALlE, P. U., PhD thesis, Université de Mons, 1994.

[18] DUMONT, R., Nuclear Fusion 49 (2009) 075033.

[19] VILlARD, L., BRUNNER, S., and VACLAVIK, J., Nuclear Fusion 35 (1995) 1173.

[20] JAEGER, E. F., BERRY, L. A., D'AZEVEDO, E., BATCHELOR, D. B., and CARTER, M. D., Physics of Plasmas 8 (2001) 1573.

[21] BRAmBILla, M. and BILATO, R., Nuclear Fusion 46 (2006) S387.

[22] EHST, D. and KARNEY, C., Nuclear Fusion 31 (1991) 1933.

[23] BONOLI, P. T., AIP Conference Proceedings 289 (1994) 192.

[24] BIlato, R., BRAmBilla, M., PAVlEnKO, I., and MEO, F., Nuclear Fusion 42 (2002) 1085.

[25] FABLE, E., Plasma Physics and Controlled Fusion 57 (2015) 045007.

[26] BRAMBILlA, M. and BIlATO, R., Nuclear Fusion 49 (2009) 085004.

[27] BIlato, R., MAJ, O., and BRAmBILlA, M., Advances in Computational Mathematics 40 (2014) 1159.

[28] Wright, J. C., VAleO, E. J., Phillips, C. K., BOnOli, P. T., and BRAMBilla, M., Communincations in Computational Physics 4 (2008) 545.

[29] BILATO, R., MAJ, O., and ANGIONI, C., Nuclear Fusion 54 (2014) 072003.

[30] Angioni, C., MCDermott, R. M., CASSON, F. J., et al., Phys. Rev. Lett. 107 (2011) 215003. 
[31] BILATO, R., BRAmBILlA, M., MAJ, O., et al., Nuclear Fusion 51 (2011) 103034.

[32] BILATO, R. and BRAMBILlA, M., AIP Conference Proceedings 1580 (2014) 306. 